\title{
Estratégias bioclimáticas em avaliação de desempenho térmico nas habitações de interesse social em Sinop/MT
}

Bioclimatic strategies in the evaluation of thermal performance in low cost housing in Sinop - MT

\author{
Cristiane Marafon', Mirian Laco ${ }^{2}$, João Carlos Machado Sanches³ ${ }^{3}$ Marlon Leão ${ }^{4}$, Érika Fernanda Toledo \\ Borges Leão 5 \\ 1,2,3,45 Universidade do Estado de Mato Grosso - UNEMAT, Brasil \\ Universidade do Estado de Mato Grosso - UNEMAT, Faculdade de Ciências Exatas e Tecnológicas \\ Departamento de Engenharia Civil
}

\begin{abstract}
Resumo
Este trabalho tem como objetivo comparar as recomendações propostas pela NBR 15220 e pelo Método de Mahoney Nebuloso com o que tem sido executado em Sinop/MT e, assim, verificar o alcance das estratégias bioclimáticas nos empreendimentos de habitações de interesse social nesse município. A metodologia adotada consistiu na verificação das características referentes às dimensões e aos materiais empregados nas Habitações de Interesse Social executadas no município. Para isso, foram objetos de estudo três exemplares de edificações residenciais unifamiliares em três empreendimentos diferentes. Os resultados demonstram que essas habitações não são executadas com vistas ao conforto térmico. Apenas uma das recomendações contidas nos métodos adotados foi plenamente atendida e, ainda, em apenas duas das três residências avaliadas. Fica ainda evidente a necessidade de adoção de aberturas maiores nas edificações, melhor orientação e distribuição dos lotes nos loteamentos, além do aumento de áreas verdes nos mesmos.
\end{abstract}

Palavras-chave: Estratégias bioclimáticas, Análise prescritiva, Método de Mahoney Nebuloso, Habitação de interesse social.

\begin{abstract}
This research aims to compare the recommendations proposed by the NBR 15220 and by the Fuzzy Mahoney's Method with what has been implemented in Sinop/MT and, thereby, verify the reach of the bioclimatic strategies in social housing projects in this city. The adopted methodology consisted of verifying the characteristics related to the dimensions and the materials employed in low cost housing executed in the city. For this, three units of residential buildings single-family from three different housing constructions were objects of study. The results demonstrate that these units are not executed with a view to thermal comfort. Only one of the recommendations contained in the methods adopted was fully met, in only two of the three residences evaluated. There is a clear need for adoption of wider openings in the buildings, better orientation and distribution of land occupation, besides the increase of green areas in them.
\end{abstract}

Keywords: Bioclimatic strategies, Prescriptive Analysis, Fuzzy Mahoney’s Method, Low cost housing. 


\section{INTRODUÇÃO}

O número de Habitações de Interesse Social (HIS) no Brasil tem crescido consideravelmente nos últimos anos. O aumento no número de empreendimentos dessa natureza deve-se ao déficit habitacional existente no país, que é de aproximadamente 5,4 milhões de unidades (IPEA, 2013). Buscando diminuir essa demanda, o governo brasileiro vem desenvolvendo diversos programas habitacionais e, por meio destes, tem atendido, sobretudo, a população considerada de baixa renda, com rendimento de até três salários mínimos.

No entanto, tal expansão tem ocorrido - quase sempre - apenas no sentido quantitativo e não qualitativo. Observa-se, ainda, a replicação de um padrão de conjuntos habitacionais em todo o território nacional, sendo que as diversidades regionais (sejam elas climáticas, culturais ou de disponibilidade de materiais e de mão-de-obra qualificada) são deixadas de lado. Assim, os conjuntos executados acabam por apresentar deficiências, dentre as quais se destacam as más condições de conforto ofertadas aos seus usuários. Tal afirmação foi comprovada por diversos autores, tais como Leão (2006), Rotta (2009) e Menezes (2006), por exemplo.

Por outro lado, muitos estudos têm sido desenvolvidos com a finalidade de adequar essas habitações ao clima onde se inserem. Através deles, foram desenvolvidas algumas normativas que apresentam métodos e recomendações a fim de propiciar maior conforto aos habitantes - seja ele térmico, lumínico, acústico, etc. Especificamente em relação ao conforto térmico, a Associação Brasileira de Normas Técnicas (ABNT) publicou, no ano de 2005, a NBR 15220 - Desempenho térmico de edificações, que traz conceitos, parâmetros, métodos de cálculo e recomendações visando à avaliação e melhoria do desempenho térmico das edificações.

Da mesma forma, outros métodos contribuem com a adequação dos espaços construídos ao clima regional. Dentre eles destaca-se o Método de Mahoney Nebuloso, que através de informações referentes à temperatura, umidade relativa do ar e pluviosidade, resulta em recomendações a serem empregadas ainda durante a fase de projeto desses empreendimentos.

Diante do exposto, tem-se como objetivo avaliar a adequação climática dos empreendimentos de habitação de interesse social na cidade de Sinop, norte do Estado de Mato Grosso, área de transição entre os biomas do Cerrado e da Amazônia, caracterizada por clima quente e úmido, com estação seca definida. Busca-se, ainda, analisar os elementos técnico-construtivos das unidades habitacionais, com relação aos parâmetros de conforto térmico vigentes na legislação brasileira, e as estratégias bioclimáticas empregadas nesses conjuntos, de acordo com as estratégias estipuladas para as condições climáticas encontradas.

\section{METODOLOGIA}

\section{I ÁREA DE ESTUDO}

A metodologia adotada consistiu na verificação das características referentes às dimensões e aos materiais empregados nas Habitações de Interesse Social executadas nessa cidade, tendo como referência a NBR 15220 e as recomendações resultantes da aplicação do Método de Mahoney Nebuloso. Os itens avaliados fazem referência, principalmente, à envoltória das edificações (paredes e cobertura) e às aberturas. Para isso, foram objetos de estudo três conjuntos residenciais unifamiliares no município de Sinop.

A cidade de Sinop está localizada na região norte do estado de Mato Grosso, mais precisamente nas coordenadas $11,86^{\circ} \mathrm{S}$ e $55,5^{\circ} \mathrm{W}$, com população estimada em 123 mil habitantes (IBGE, 2013).

Segundo a classificação climática apresentada no Zoneamento Socioeconômico-ecológico de Mato Grosso, o estado de Mato Grosso apresenta duas unidades climáticas: I - Clima Equatorial Continental com Estação Seca Definida (3 a 5 meses); e II - Clima Tropical Continental Alternadamente Úmido e Seco. Essas unidades são, ainda, divididas em subunidades de acordo com características referentes à temperatura, pluviosidade e altitude. (MAITELLI, 2005). Segundo essa classificação, o município de Sinop encontra-se nos domínios da subunidade I-B, apresentando, assim, temperaturas médias anuais entre $24,3^{\circ}$ e $26,8^{\circ} \mathrm{C}$, além de total pluviométrico entre $1800 \mathrm{~mm}$ e $2200 \mathrm{~mm}$, com período de seca nos meses de abril a setembro.

$\mathrm{Na}$ escolha das residências estudadas, procurou-se compor uma amostra característica das HIS 
no município. No entanto, devido a não disponibilização dos dados por parte das empresas responsáveis, foram descartados dois loteamentos dos cinco inicialmente previstos. Assim, foram selecionadas três residências, denominadas genericamente de $\mathrm{A}, \mathrm{B}$ e $\mathrm{C}$, que representam as tipologias encontradas nos empreendimentos selecionados (compostos de unidades idênticas posicionadas no centro dos lotes).

As habitações escolhidas apresentaram semelhanças com relação ao número de cômodos, sendo constituídas por dois quartos, sala, cozinha e banheiro. As diferenças estão nas áreas dos cômodos. Assim, as unidades habitacionais (UH) apresentam áreas entre 30,6 m2 e $52 \mathrm{~m} 2$. A identificação das UH é apresentada no Quadro 1.

Quadro 1- Identificação das Residências

\begin{tabular}{|c|c|c|c|c|}
\hline Residência & Residencial & Construtora Responsável & $\begin{array}{c}\text { Público alvo } \\
\text { (salários mínimos) }\end{array}$ & Ano de Entrega \\
\hline A & José Adriano Leitão & Sisan & $>3$ & 2010 \\
\hline B & Vila América & Pontual & $\leq 3$ & 2012 \\
\hline C & Sebastião de Matos I & Pontual & $\leq 1$ & 2012 \\
\hline
\end{tabular}

A Figura 01 traz a localização dos residenciais amostrados e as Figuras 02, 03 e 04 apresentam as fachadas das edificações estudadas.

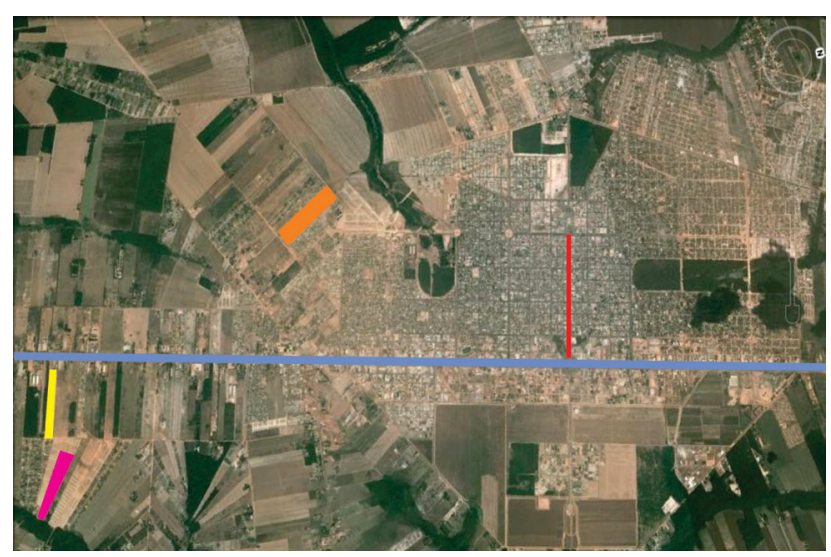

Figura 01 - Localização dos Residenciais. Amarelo: Sebastião de Matos I; Rosa: Vila América; Laranja: José Adriano Leitão; Verme1ho: Avenida Gov. Júlio Campos (área central da cidade); Azul: BR 163.

Fonte: GOOGLE EARTH, 2013 (adaptado).

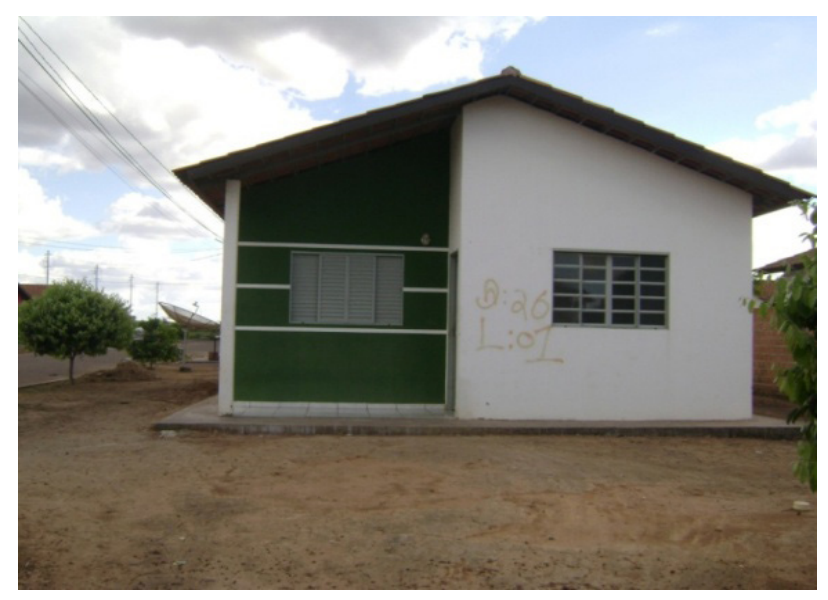

Figura 02 - Fachada residência A 


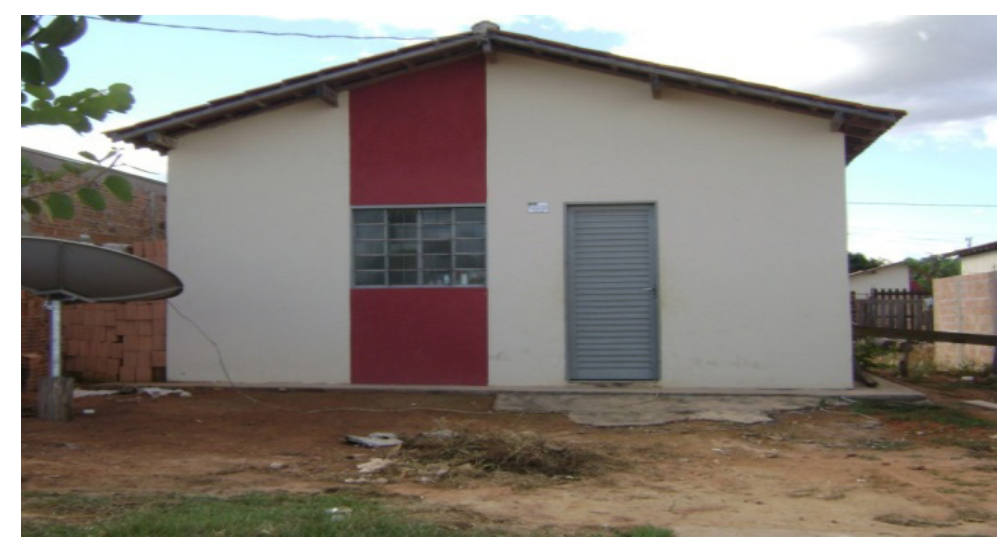

Figura 03 - Fachada residência B

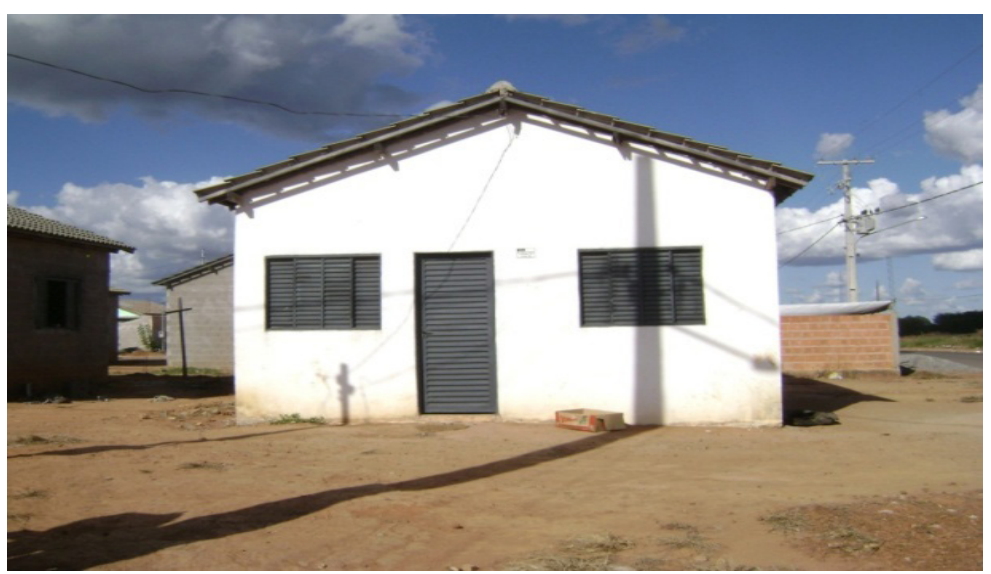

Figura 04- Fachada residência C

De forma geral, as edificações apresentaram semelhanças na distribuição espacial, como pode ser visto nas Figuras 05, 06 e 07.

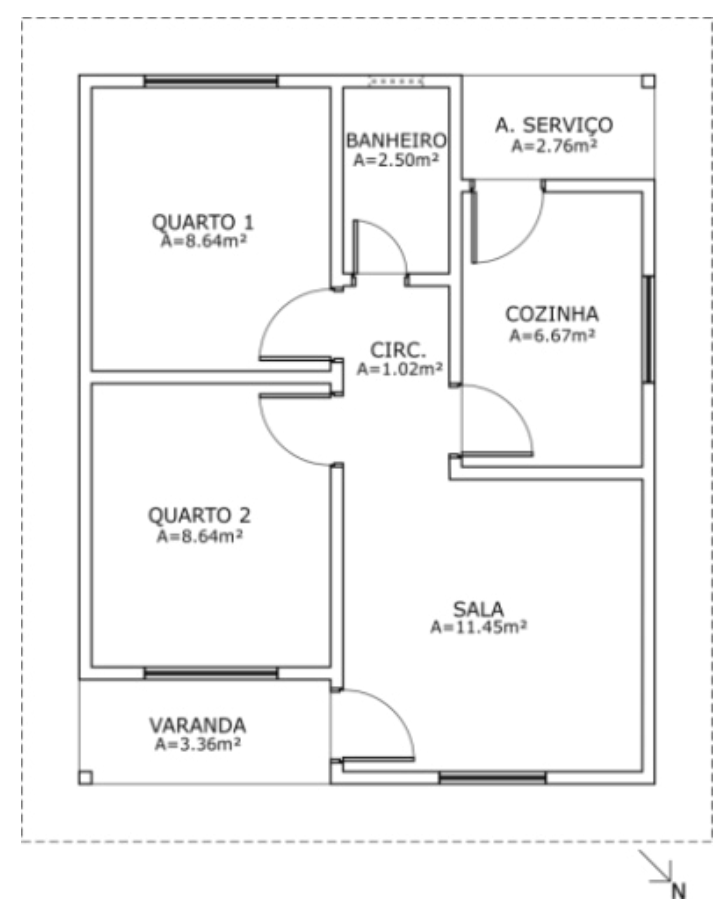

Figura 05- Planta esquemática residência A

Fonte: SISAN ENGENHARIA, 2013 (adaptado). 


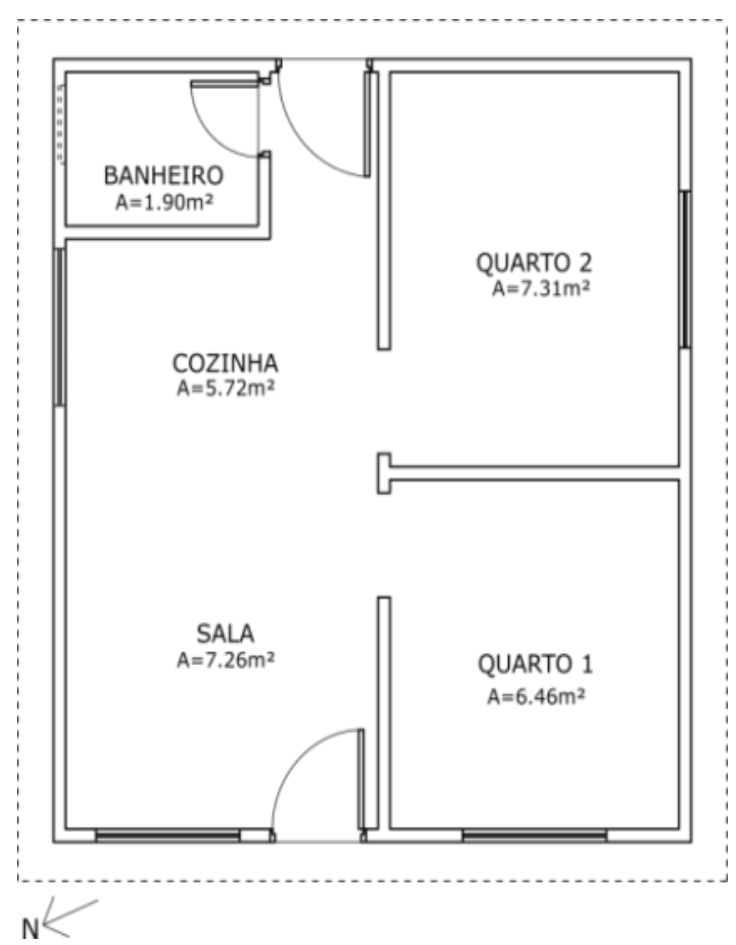

Figura 06- Planta esquemática residência $B$

Fonte: PONTUAL CONSTRUTORA, 2013 (adaptado).

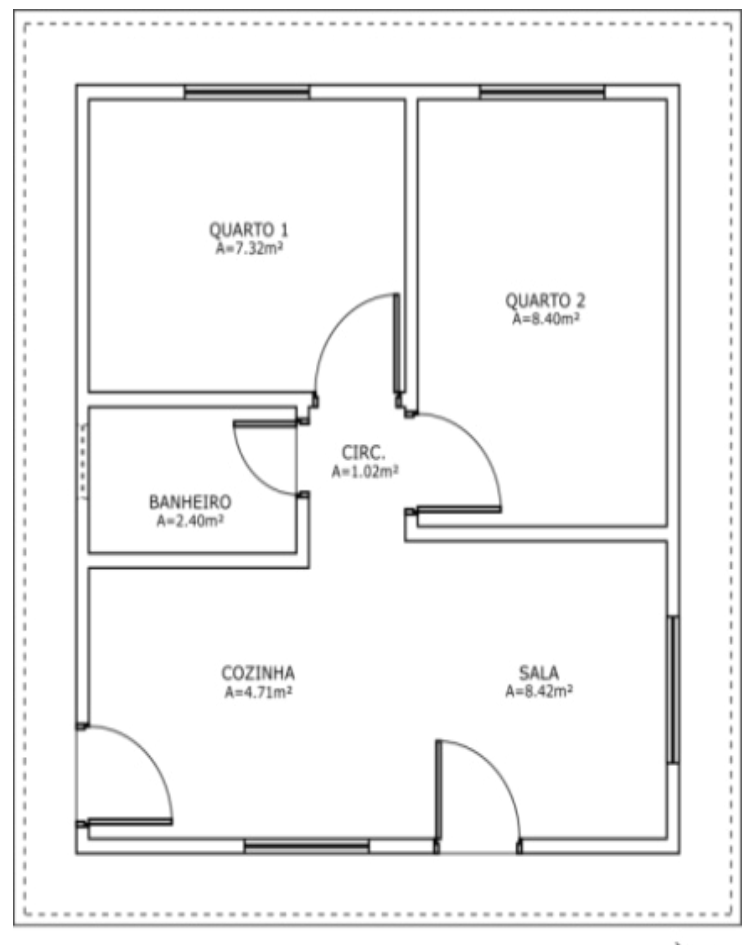

Figura 07- Planta esquemática residência $C$

Fonte: PONTUAL CONSTRUTORA, 2013 (adaptado).

\subsection{MAHONEY NEBULOSO}

O Método de Mahoney Nebuloso é uma versão, modificada por Harris (1999), dos Quadros de Mahoney, desenvolvidos por Carl Mahoney, Martin Evans e Otto Koenigsberger e publicados pelas Nações Unidas, em 1971, sob o título Climate and House Design. A publicação tinha como objetivo criar um método que adequasse a edificação ao clima da região e também visava o conforto térmico e 
o baixo custo dessas edificações, principalmente em países em desenvolvimento. (UNITED NATIONS, 1971 apud SENA, 2004).

A adoção da lógica nebulosa apresenta resultados semelhantes ao método tradicional para regiões com clima bem definido, no entanto mostrou-se mais eficaz para regiões consideradas de transição climática, como mostra Sena (2004). Esse fato constitui a principal justificativa para a aplicação da versão nebulosa na cidade de Sinop.

O processo de determinação das recomendações do Método Nebuloso para a cidade de Sinop foi realizado por meio da análise de dados climáticos obtidos junto ao Instituto Nacional de Meteorologia - INMET. Foram analisados dados horários, referentes ao período compreendido entre os anos de 2007 e 2011, que resultaram nas recomendações encontradas no item 4.1.

\subsection{ANÁlISE DE ACORDO COM A NBR I 5220}

A análise prescritiva foi realizada empregando-se as disposições da NBR 15220 (ABNT, 2003). Segundo o Zoneamento Bioclimático Brasileiro (ZBBR), que constitui a parte 3 da referida norma, pode-se considerar o município de Sinop como pertencente à zona bioclimática 5 . Dessa forma, para as Habitações de Interesse Social executadas nesse município, a norma recomenda o uso de aberturas para ventilação médias, o sombreamento das aberturas, a utilização de paredes leves refletoras e de coberturas leves e isoladas.

Além destas recomendações, a norma sugere estratégias de condicionamento térmico passivo para os períodos de inverno e verão, que consistem na adoção de paredes internas pesadas e o uso de ventilação cruzada, respectivamente.

A definição do tipo de envoltória (leve refletora, isolada) é feita através da determinação dos valores referentes à Transmitância Térmica (U), Atraso Térmico (j) e Fator Solar (FS0) dos elementos da envoltória.

\subsection{Coleta de dados}

Para a análise realizada, os dados foram coletados junto às construtoras responsáveis pelos conjuntos residenciais. Dessa forma, foram processadas informações provenientes de plantas, memoriais descritivos, entrevistas com os responsáveis, observações in loco, entre outras fontes.

Quanto às edificações em si, foi possível determinar as áreas de piso, aberturas para ventilação, orientação das fachadas, entre outros aspectos. Através destes, foram obtidos dados referentes aos materiais empregados, tais como espessura, condutividade térmica, massa específica, absortância e demais informações pertinentes.

Com os dados em mãos e empregando-se os conceitos e procedimentos indicados, foi possível, então, mensurar as características das edificações e compará-las com os valores indicados na NBR 15220 e no método de Mahoney Nebuloso.

\section{RESULTADOS E DISCUSSÕES}

\section{I ANÁlise pelo método de Mahoney nebuloso}

Com os dados horários referentes ao período em questão, foi possível determinar as temperaturas médias (mínimas e máximas), a amplitude térmica, a umidade relativa do ar média e a pluviosidade média para cada mês em cada ano. Assim, obteve-se uma média mensal de todos os anos compreendidos no período mencionado. Com a obtenção de tais valores foi possível, então, determinar as suas respectivas pertinências (m). Os procedimentos adotados resultaram nos dados apresentados no Quadro 2.

A partir desses valores, verificou-se a presença de dois conjuntos Bem Estar para o clima de Sinop, o Conjunto 1 e o Conjunto 4. Nas Figuras 08, 09 e 10 pode-se verificar o resultado da aplicação do método nos conjuntos Bem Estar (BE), Confortável e na determinação dos Rigores Térmicos (RT) para os meses de Janeiro e Junho (escolhidos como exemplo por representarem os períodos chuvoso e seco, respectivamente). Outros gráficos foram gerados para determinar esses conjuntos para todos os meses do ano. 
Quadro 2 - Resumo de pertinências

\begin{tabular}{|c|c|c|c|c|c|c|c|c|c|c|c|c|}
\hline $\begin{array}{c}\text { DADOS/ } \\
\text { MÊS }\end{array}$ & Jan & Fev & Mar & Abr & Mai & Jun & Jul & Ago & Set & Out & Nov & Dez \\
\hline Temp. Máx. (Média ${ }^{\circ} \mathrm{C}$ ) & 30,07 & 30,50 & 30,89 & 31,48 & 30,35 & 32,61 & 32,65 & 29,57 & 35,14 & 33,26 & 31,39 & 30,11 \\
\hline Temp. Mín. (Média $\left.{ }^{\circ} \mathrm{C}\right)$ & 21,47 & 21,48 & 21,35 & 21,09 & 19,09 & 18,99 & 18,23 & 15,19 & 19,58 & 21,47 & 21,63 & 21,40 \\
\hline Temp. Média $\left({ }^{\circ} \mathrm{C}\right)$ & 25,77 & 25,99 & 26,12 & 26,28 & 24,72 & 25,80 & 25,44 & 22,38 & 27,36 & 27,37 & 26,51 & 25,76 \\
\hline$\mu_{\text {TM-Alta }}$ & 1,0 & 1,0 & 1,0 & 1,0 & 1,0 & 1,0 & 1,0 & 1,0 & 1,0 & 1,0 & 1,0 & 1,0 \\
\hline Amp. Térmica $\left({ }^{\circ} \mathrm{C}\right)$ & 8,59 & 9,02 & 9,54 & 10,39 & 11,26 & 13,62 & 14,42 & 14,38 & 15,56 & 11,79 & 9,76 & 8,71 \\
\hline$\mu_{\text {AT-Pequena }}$ & 0,57 & 0,55 & 0,53 & 0,49 & 0,47 & 0,41 & 0,37 & 0,37 & 0,30 & 0,46 & 0,51 & 0,56 \\
\hline$\mu_{\text {AT-Grande }}$ & 0,43 & 0,45 & 0,48 & 0,51 & 0,53 & 0,59 & 0,63 & 0,63 & 0,70 & 0,54 & 0,49 & 0,44 \\
\hline Umid. Rel. Média (\%) & 80,29 & 78,72 & 75,83 & 75,53 & 65,22 & 57,04 & 50,04 & 40,61 & 54,25 & 68,27 & 75,29 & 79,36 \\
\hline$\mu_{\text {URM-Seco }}$ & 0,10 & 0,15 & 0,27 & 0,28 & 0,55 & 0,63 & 0,70 & 0,79 & 0,66 & 0,52 & 0,29 & 0,13 \\
\hline$\mu_{\text {URM-Úmido }}$ & 0,90 & 0,85 & 0,73 & 0,72 & 0,45 & 0,37 & 0,30 & 0,21 & 0,34 & 0,48 & 0,71 & 0,87 \\
\hline Pluviosidade $(\mathrm{mm})$ & 219,2 & 213,8 & 143,9 & 71,6 & 23,64 & 5,8 & 5,32 & 6,32 & 59,4 & 165,4 & 248,9 & 261,8 \\
\hline$\mu_{\text {PL-Alta }}$ & 0,49 & 0,48 & 0,32 & 0,16 & 0,05 & 0,01 & 0,01 & 0,01 & 0,13 & 0,37 & 0,56 & 0,59 \\
\hline
\end{tabular}
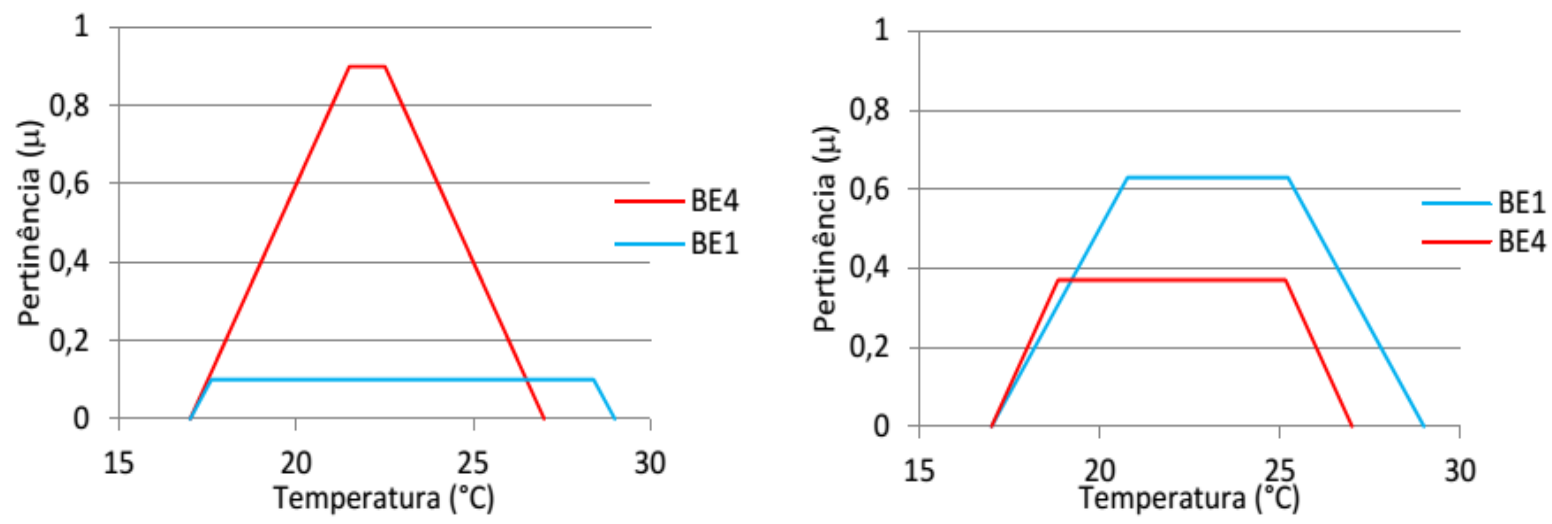

Figura 08- Conjuntos bem estar janeiro e junho
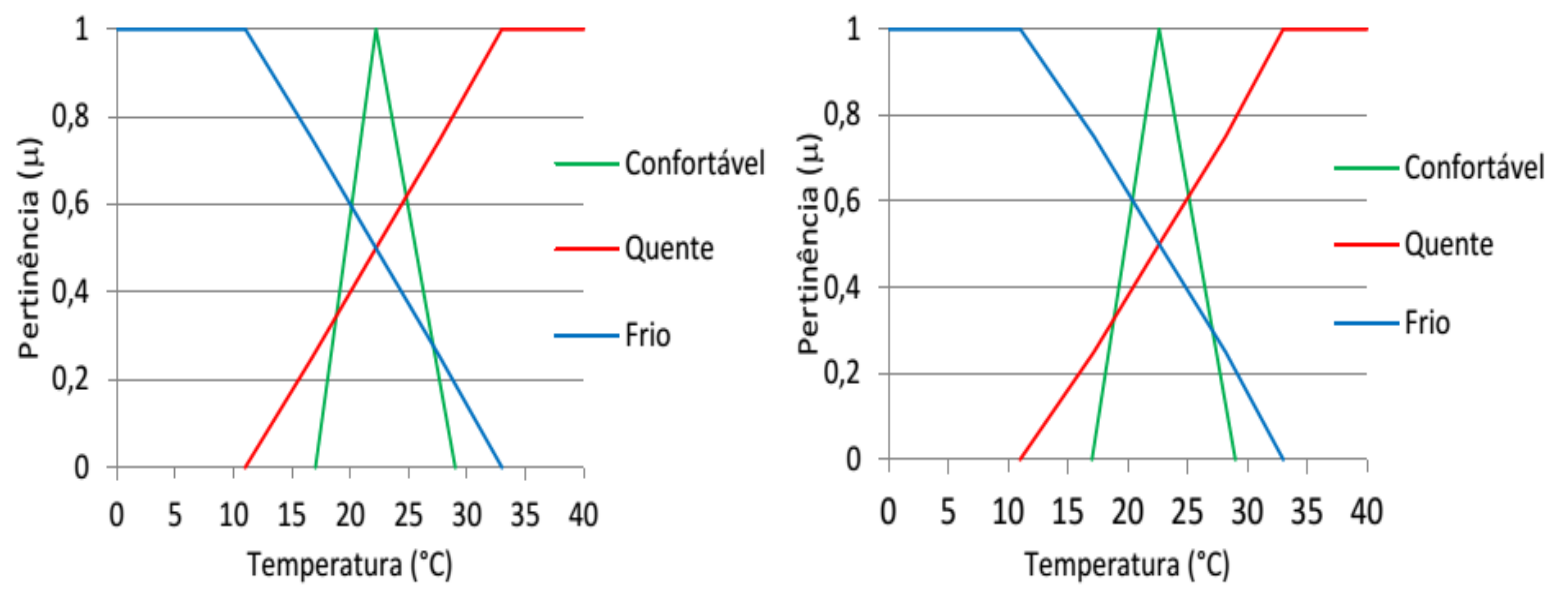

Figura 09- Conjunto confortável janeiro e junho 

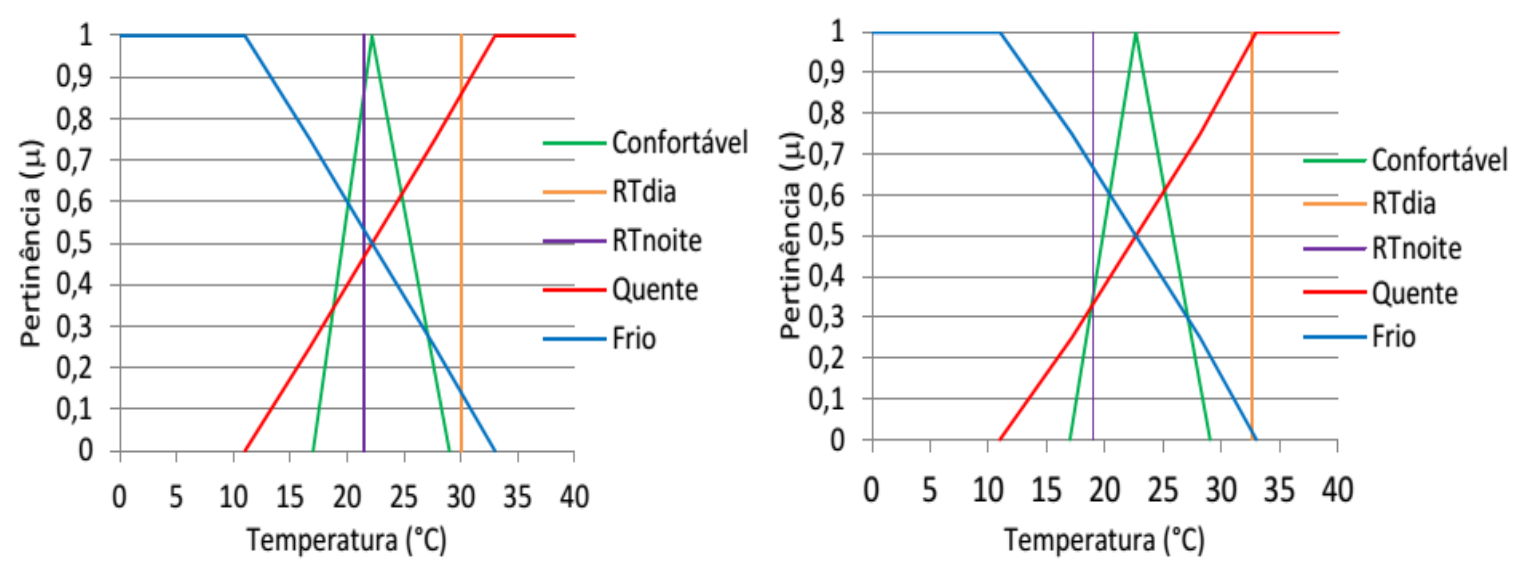

Figura 10- Rigores térmicos janeiro e junho

Realizados os procedimentos anteriores, pode-se, então, calcular as pertinências referentes a cada um dos indicadores e, através delas, determinar a frequência anual (fanual) de cada indicador. $\mathrm{O}$ Quadro 3 apresenta os valores obtidos.

Os valores das frequências dos indicadores foram inseridos nos gráficos, indicados pela metodologia empregada, e a combinação das frequências encontradas resultou nas pertinências, indicadas no Quadro 4, e suas consequentes recomendações de projeto.

Quadro 3 - Pertinências dos indicadores

\begin{tabular}{|c|c|c|c|c|c|c|c|c|c|c|c|c|c|}
\hline INDICADORES & JAN & FEV & MAR & ABR & MAI & JUN & JUL & AGO & SET & OUT & NOV & DEZ & $\mathbf{f}_{\text {anual }}$ \\
\hline$\mu_{\mathrm{H} 1}$ & 0,86 & 0,85 & 0,73 & 0,72 & 0,47 & 0,41 & 0,37 & 0,37 & 0,34 & 0,48 & 0,71 & 0,86 & 7,17 \\
\hline$\mu_{\mathrm{H} 2}$ & - & - & - & - & - & - & - & - & - & - & - & - & - \\
\hline$\mu_{\mathrm{H} 3}$ & 0,49 & 0,48 & 0,32 & 0,16 & 0,05 & 0,01 & 0,01 & 0,01 & 0,13 & 0,37 & 0,56 & 0,59 & 3,18 \\
\hline$\mu_{\mathrm{A} 1}$ & 0,10 & 0,15 & 0,27 & 0,28 & 0,53 & 0,59 & 0,63 & 0,63 & 0,66 & 0,52 & 0,29 & 0,13 & 4,78 \\
\hline$\mu_{\mathrm{A} 2}$ & 0,10 & 0,15 & 0,27 & 0,28 & 0,37 & 0,35 & 0,30 & 0,63 & 0,46 & 0,52 & 0,29 & 0,13 & 3,85 \\
\hline$\mu_{\mathrm{A} 3}$ & 0,14 & 0,12 & 0,10 & 0,08 & 0,14 & 0,02 & 0,02 & 0,18 & - & - & 0,08 & 0,14 & 1,02 \\
\hline
\end{tabular}

Quadro 4 - Recomendações do Método de Mahoney Nebuloso para Sinop

\begin{tabular}{|c|c|c|c|}
\hline R1 & $\begin{array}{c}\text { Edifícios orientados sobre o eixo L-O para } \\
\text { reduzir a exposição ao sol }\end{array}$ & R13 & Paredes pesadas, interiores e exteriores \\
\hline R4 & $\begin{array}{c}\text { Espaço aberto para penetração de brisa, } \\
\text { porém com proteção do vento quente ou frio }\end{array}$ & R14 & Coberturas isoladas leves \\
\hline R6 & $\begin{array}{c}\text { Habitações em fileira única. Dispositivo } \\
\text { permanente para o movimento do ar }\end{array}$ & R16 & Espaço necessário para dormir ao ar livre \\
\hline R11 & Aberturas medianas, $20 \%-40 \%$ & R17 & Necessidade de proteção contra a chuva intensa \\
\hline
\end{tabular}

A recomendação referente à distribuição/orientação da edificação (R1) propõe que a habitação tenha seu eixo longitudinal orientado na direção Leste-Oeste, a fim de reduzir a exposição ao sol. Essa orientação garante que os ambientes de longa permanência tenham suas aberturas posicionadas nas fachadas Norte-Sul. Na fachada Sul, tem-se a menor insolação para a latitude em questão $\left(11,86^{\circ} \mathrm{S}\right)$. Já a fachada Norte deve ser protegida com elementos de proteção solar, de modo a proteger as abertu- 
ras, principalmente nas horas próximas ao meio-dia, quando a intensidade da radiação solar é maior.

Esta recomendação está ligada à distribuição/orientação dos residenciais como um todo. Sendo assim, existem unidades que estão de acordo com tal recomendação e unidades que não estão de acordo.

Com relação ao espaçamento e movimento do ar (R4), recomenda-se que haja espaço para penetração de brisa, mas que se verifiquem mecanismos de proteção contra o vento seco (carregado de partículas) ou frio.

As residências estudadas não apresentaram mecanismos de proteção contra o vento. Quando se analisa os residenciais como um todo, percebe-se que o espaçamento entre as habitações, na maioria das vezes, não proporciona a movimentação do ar. Além disso, a vegetação, que atuaria na proteção das residências, é escassa. Verifica-se maior número de árvores no Residencial José Adriano Leitão, entregue há mais tempo, indicando uma preocupação por parte dos moradores quanto a este quesito.

Com base no Método de Mahoney Nebuloso, as aberturas devem apresentar dimensões médias (R11), representando de $25 \%$ a $40 \%$ da área das paredes norte e sul. Desse modo, é possível aproveitar a incidência dos ventos predominantes na região, a partir da ventilação cruzada nos ambientes. O que se verificou, no entanto, foi o não cumprimento deste quesito em todas as edificações. A residência A apresentou valor insuficiente para uma das fachadas e sequer apresentou aberturas na outra. As demais residências também apresentaram valores bem abaixo do estipulado.

A recomendação do método para as paredes (R13), tanto internas quanto externas, é que as mesmas sejam pesadas, apresentando alta capacidade térmica. Dessa forma, o calor externo terá maior dificuldade para chegar ao ambiente interno e, quando isso ocorrer, o mesmo estará mais abrandado. Esta recomendação só não foi alcançada, principalmente, devido ao baixo atraso térmico apresentado pelas paredes das edificações.

Segundo o Método, a cobertura a ser empregada deve ser leve, porém bem isolada (R14). No entanto não foram verificadas tais características nas habitações estudadas. A residência A apresentou cobertura executada em telhas cerâmicas e é a única que teve a presença de laje. A Residência B apresentou telha cerâmica e forro em PVC e a residência $C$ apresentou telhas de concreto sem a presença de qualquer forro.

A recomendação que indica a necessidade de um espaço adequado para dormir ao ar livre (R16) não foi atendida em nenhuma das residências.

Já a recomendação de proteção contra a chuva (R17) é atendida quando se observa a existência de beirais mais largos, varandas e áreas cobertas. Apenas a residência A apresentou varanda. As demais residências não apresentam tais estruturas e os beirais não são suficientes. A residência $C$ é a que apresenta o estado mais crítico, com beirais quase inexistentes.

\subsection{ANÁLISE PRESCRITIVA PELA NBR I 5220}

A transmitância térmica (U) foi determinada para a envoltória da edificação. Assim, obtiveram-se dois valores de transmitância térmica, um para as paredes e outro para a cobertura. Para o cálculo da transmitância térmica empregou-se a Equação (1).

Nessa equação, RT representa a resistência térmica total do elemento (parede ou cobertura) e

$$
\mathrm{U}=\frac{1}{\mathrm{R}_{\mathrm{T}}}
$$

foi obtida por meio da soma das resistências individuais dos componentes do elemento em questão. Para cada componente, a resistência térmica foi calculada conforme a Equação (2), abaixo:

$$
\mathrm{R}=\frac{\mathrm{e}}{\lambda}
$$

Onde:

e: espessura (mm)

$\lambda$ : condutividade térmica (W/m.K) do componente, dada pelo Quadro 5.

Compuseram, ainda, a resistência térmica total, as resistências térmicas superficiais internas $\left(\mathrm{R}_{\mathrm{si}}\right)$ e externas $\left(\mathrm{R}_{\mathrm{se}}\right)$, que foram obtidas no Quadro 6. 
Também foi necessário considerar a resistência térmica das câmaras de ar (Rar),, dada pelo Quadro 7.

Quadro 5 - Densidade de massa aparente (r), condutividade térmica (l) e calor específico (c) de materiais (ABNT, 2005)

\begin{tabular}{|c|c|c|c|}
\hline Material & $\boldsymbol{\rho}\left(\mathbf{k g} / \mathbf{m}^{\mathbf{3}}\right)$ & $\boldsymbol{\lambda} \mathbf{( W / m . K )}$ & $\mathbf{c}(\mathbf{k J} / \mathbf{( k g . K )}$ \\
\hline Argamassa & 2000 & 1,15 & 1,00 \\
\hline Cerâmica (telha) & 2000 & 1,05 & 0,92 \\
\hline Cerâmica (tijolo) & 1600 & 0,90 & 0,92 \\
\hline Concreto (laje) & 2200 & 1,75 & 1,00 \\
\hline Concreto (telha) & 2200 & 1,75 & 1,00 \\
\hline Policloreto de vinila (PVC) & 1300 & 0,20 & 0,96 \\
\hline
\end{tabular}

Quadro 6 - Resistência térmica superficial interna e externa (ABNT, 2005)

\begin{tabular}{|c|c|c|c|c|c|}
\hline \multicolumn{3}{|c|}{$\mathrm{R}_{\mathrm{si}}\left(\mathrm{m}^{2} . \mathrm{K}\right) / \mathrm{W}$} & \multicolumn{3}{c|}{$\mathrm{R}_{\mathrm{se}}\left(\mathrm{m}^{2} . \mathrm{K}\right) / \mathrm{W}$} \\
\hline \multicolumn{2}{|c|}{ Direção do fluxo de calor } & \multicolumn{3}{c|}{ Direção do fluxo de calor } \\
\hline & $\boldsymbol{1}$ & - & & $\boldsymbol{1}$ & \\
\hline 0,13 & 0,10 & 0,17 & 0,04 & 0,04 & 0,04 \\
\hline
\end{tabular}

Quadro 7 - Resistência térmica de câmaras de ar não ventiladas (ABNT, 2005)

\begin{tabular}{|c|c|c|c|c|}
\hline \multirow{2}{*}{ Natureza da superfície da câmara de ar Espessura da câmara de ar $(\mathrm{cm})$} & \multicolumn{3}{|c|}{$\mathrm{R}_{\mathrm{ar}}\left(\mathrm{m}^{2} . \mathrm{K}\right) / \mathrm{W}$} \\
\cline { 3 - 6 } & & Direção do fluxo de calor \\
\hline \multirow{2}{*}{$\begin{array}{c}\text { Superfície de alta emissividade } \\
(\mathrm{e} \geq 0,8)\end{array}$} & $1,0 \leq \mathrm{e} \leq 2,0$ & 0,14 & 0,13 & 0,15 \\
\cline { 3 - 6 } & $2,0 \leq \mathrm{e} \leq 5,0$ & 0,16 & 0,14 & 0,18 \\
\cline { 3 - 6 } & $\mathrm{e}>5,0$ & 0,17 & 0,14 & 0,21 \\
\hline \multirow{2}{*}{$\begin{array}{c}\text { Superfície de baixa emissividade } \\
(\mathrm{e} \leq 0,2)\end{array}$} & $1,0 \leq \mathrm{e} \leq 2,0$ & 0,29 & 0,23 & 0,29 \\
\cline { 2 - 6 } & $2,0 \leq \mathrm{e} \leq 5,0$ & 0,37 & 0,25 & 0,43 \\
\cline { 2 - 6 } & $\mathrm{e}>5,0$ & 0,34 & 0,27 & 0,61 \\
\hline
\end{tabular}

As paredes das residências são compostas por tijolos de 8 furos $(9 \mathrm{~cm} \times 19 \mathrm{~cm} \times 19 \mathrm{~cm})$. A espessura da argamassa de revestimento adotada pelas empresas é de, no máximo, $2,5 \mathrm{~cm}$. A argamassa de assentamento, por sua vez, não ultrapassa $1,0 \mathrm{~cm}$ de espessura.

Para o cálculo da transmitância térmica das paredes, o fluxo considerado ocorre no sentido horizontal, considerando as seções das residências A e B, compostas por tijolos assentados na menor dimensão, com reboco de ambos os lados, resultando em $\mathrm{U}=2,33 \mathrm{~W} /\left(\mathrm{m}^{2} . \mathrm{K}\right)$. Já a residência $\mathrm{C}$ apresenta tijolos assentados na menor dimensão, com reboco apenas na superfície externa, resultando em $\mathrm{U}=4,12 \mathrm{~W} /\left(\mathrm{m}^{2} \cdot \mathrm{K}\right)$.

Para o cálculo da transmitância térmica da cobertura, a direção do fluxo adotada foi descendente. Assim, para a residência A tem-se $U=2,16 \mathrm{~W} /\left(\mathrm{m}^{2} . \mathrm{K}\right)$, para a residência $B, U=2,09 \mathrm{~W} /\left(\mathrm{m}^{2} . \mathrm{K}\right)$, e, para a residência $C, U=4,64 \mathrm{~W} /\left(\mathrm{m}^{2} . \mathrm{K}\right)$.

Os resultados obtidos expressam a diferença entre os métodos construtivos adotados para as coberturas. A residência A apresenta telha cerâmica e laje de concreto. A residência B apresenta forro 
em PVC e telha cerâmica. A residência C, por sua vez, apresenta telhado composto por telhas de concreto e não há forro.

Para o cálculo do atraso térmico utilizou-se a Equação (3), abaixo:

$$
\varphi=1,382 \cdot \mathrm{R}_{\mathrm{t}} \cdot \sqrt{\mathrm{B}_{1}+\mathrm{B}_{2}}
$$

Onde as variáveis B1 e B2 podem ser obtidas empregando-se as Equações (4), (5) e (6), a seguir.

$$
B_{1}=0,226 \cdot \frac{B_{0}}{R_{t}}
$$

$$
B_{2}=0,205 \cdot\left(\frac{(\lambda \cdot \rho \cdot c)_{\text {ext }}}{R_{t}}\right) \cdot\left(R_{\text {ext }}-\frac{R_{e}-R_{\text {ext }}}{10}\right)
$$

$$
B_{0}=C_{T}-C_{\text {Text }}
$$

Para as paredes, o cálculo do atraso térmico resultou em $\mathrm{j}=3,04$ horas para as residências A e $B$ e em $\mathrm{j}=0,95$ horas para a residência $\mathrm{C}$.

A semelhança verificada entre os valores de atraso térmico ocorreu pela mesma razão que a transmitância térmica, ou seja, semelhança nos métodos construtivos das paredes. Novamente, a residência $\mathrm{C}$ se diferencia devido à inexistência de revestimento interno e por apresentar unicamente o chapisco como revestimento externo, na maior extensão do seu perímetro.

Já com relação às coberturas, os valores obtidos para o atraso térmico foram $j=5,14$ horas para a residência $A, j=1,61$ horas para a residência $B$ e $j=1,46$ horas para a residência $C$.

No cálculo do fator solar utiliza-se a Equação (7), a seguir.

$$
\mathrm{FS}_{0}=4 \cdot \mathrm{U} \cdot \alpha
$$

Onde a é a absortância do material, dada pelo Quadro 8. 
Quadro 8 - Emissividade (e) e absortância (a) para diferentes superfícies (ABNT, 2005)

\begin{tabular}{|c|c|c|}
\hline Tipo de Superfície & $\boldsymbol{\alpha}$ & $\boldsymbol{\varepsilon}$ \\
\hline Caiação nova & $0,112 / 0,15$ & 0,90 \\
\hline Concreto aparente & $0,65 / 0,80$ & $0,85 / 0,95$ \\
\hline Telha cerâmica & $0,75 / 0,80$ & $0,85 / 0,95$ \\
\hline Tijolo aparente & $0,65 / 0,80$ & $0,85 / 0,95$ \\
\hline Reboco claro & $0,30 / 0,50$ & $0,85 / 0,95$ \\
\hline \multicolumn{3}{|c|}{ Pintura } \\
\hline Branca & 0,20 & 0,90 \\
\hline Amarela & 0,30 & 0,90 \\
\hline Verde clara & 0,40 & 0,90 \\
\hline Alumínio & 0,40 & 0,50 \\
\hline Verde escura & 0,70 & 0,90 \\
\hline Vermelha & 0,74 & 0,90 \\
\hline Preta & 0,97 & 0,90 \\
\hline
\end{tabular}

Para o cálculo do fator solar das paredes também foi necessário realizar o procedimento de ponderação, uma vez que todas as habitações apresentaram diferenças nas cores empregadas na pintura de suas fachadas.

Desse modo, os valores de fator solar obtidos são apresentados na Tabela 1.

Tabela 1 - Fator solar das paredes

\begin{tabular}{ccccccc}
\hline \multirow{2}{*}{ Residência } & Cor & Área & $\boldsymbol{\alpha}$ & Área pond. & $\begin{array}{c}\boldsymbol{\alpha} \\
\text { pond. }\end{array}$ & FS $_{\mathbf{0}}$ (\%) \\
\hline \multirow{3}{*}{$\mathbf{A}$} & Branco & 90,64 & 0,20 & 0,92 & 0,184 & \\
& Verde escuro & 8,39 & 0,70 & 0,08 & 0,056 & $\mathbf{2}, \mathbf{2 4}$ \\
& Total & $\mathbf{9 9 , 0 3}$ & --- & $\mathbf{1 , 0 0}$ & $\mathbf{0 , 2 4 0}$ & \\
\hline \multirow{2}{*}{$\mathbf{B}$} & Amarelo & 66,40 & 0,30 & 0,95 & 0,285 & \\
& Vermelho & 3,30 & 0,74 & 0,05 & 0,037 & $\mathbf{3}, \mathbf{0 0}$ \\
& Total & $\mathbf{6 9 , 7 0}$ & --- & $\mathbf{1 , 0 0}$ & $\mathbf{0 , 3 2 2}$ & \\
\hline \multirow{2}{*}{$\mathbf{C}$} & Branco & 14,04 & 0,20 & 0,23 & 0,046 & \\
& Reboco claro & 47,40 & 0,40 & 0,77 & 0,308 & $\mathbf{5 , 8 3}$ \\
& Total & $\mathbf{6 1 , 4 4}$ & -- & $\mathbf{1 , 0 0}$ & $\mathbf{0 , 3 5 4}$ & \\
\hline
\end{tabular}

Com os cálculos do fator solar da cobertura, foram obtidos os valores de $\mathrm{FS} 0=6,90 \%$ para residência $A, 6,67 \%$ para a residência $B$ e de $13,44 \%$ para a residência $C$.

Para o cálculo das áreas das aberturas para ventilação, foram considerados os cômodos tidos como de permanência prolongada, ou seja, quartos, salas e cozinhas. Além disso, devido às janelas empregadas serem do tipo "de correr", foram utilizados valores referentes à metade das áreas das mesmas, conforme indicado em CBIC (2013).

Todas as porcentagens encontradas são inferiores às recomendadas pela NBR 15220, sendo assim, insuficientes para que a ventilação seja efetiva.

\subsection{Avaliação geral}

O Quadro 9 apresenta um resumo das recomendações adotadas e não adotadas nos empreendimentos avaliados no município de Sinop. 
Quadro 9-Quadro-resumo. A: Elemento ausente; P: Elemento presente; P*: Elemento presente com ressalvas anteriormente apresentadas.

\begin{tabular}{|c|c|c|c|}
\hline RECOMENDAÇÕES & \multicolumn{3}{|c|}{ RESIDENCIAS ESTUDADAS } \\
\hline MAHONEY NEBULOSO & A & B & C \\
\hline Cobertura (isolada Leve) & $\mathrm{A}$ & $\mathrm{A}$ & $\mathrm{A}$ \\
\hline Paredes (pesadas) & $\mathrm{A}$ & $\mathrm{A}$ & $\mathrm{A}$ \\
\hline $\begin{array}{c}\text { Aberturas medianas } \\
(20 \%<\mathrm{A}<40 \%)\end{array}$ & $\mathrm{A}$ & $\mathrm{A}$ & $\mathrm{A}$ \\
\hline Proteção contra a chuva intensa & $\mathrm{P}$ & $\mathrm{A}$ & $\mathrm{A}$ \\
\hline Espaçamento e movimento do ar & $\mathrm{A}$ & $\mathrm{A}$ & $\mathrm{A}$ \\
\hline Espaço necessário para dormir ao ar livre & $\mathrm{A}$ & $\mathrm{A}$ & $\mathrm{A}$ \\
\hline Orientação sobre o eixo L-O & $\mathrm{P}$ & $\mathrm{A}$ & $\mathrm{P}$ \\
\hline NBR 15220 & $\mathrm{A}$ & $\mathrm{B}$ & $\mathrm{C}$ \\
\hline Cobertura (leve isolada) & $\mathrm{A}$ & $\mathrm{A}$ & $\mathrm{A}$ \\
\hline $\begin{array}{c}\text { Paredes externas } \\
(\text { leves refletoras) }\end{array}$ & $\mathrm{P}$ & $\mathrm{P}$ & $\mathrm{A}$ \\
\hline $\begin{array}{c}\text { Aberturas médias } \\
(15 \%<\text { A }<25 \%)\end{array}$ & $\mathrm{A}$ & $\mathrm{A}$ & $\mathrm{A}$ \\
\hline Sombreamento de Aberturas & $\mathrm{A}$ & $\mathrm{A}$ & $\mathrm{A}$ \\
\hline Paredes internas (pesadas) & $\mathrm{A}$ & $\mathrm{A}$ & $\mathrm{A}$ \\
\hline Ventilação Cruzada & $\mathrm{P}$ & $\mathrm{P}$ & $\mathrm{P}$ \\
\hline
\end{tabular}

Diante disso, percebe-se que apenas uma das recomendações foi plenamente atendida e, ainda assim, apenas nas Residências A e B. A grande maioria das recomendações foi negligenciada, sobretudo, na Residência C.

Isso evidencia a preocupação com o número de habitações construídas e não, necessariamente, com o conforto térmico por elas propiciado.

Os resultados indicam a presença de falhas relacionadas tanto com o projeto arquitetônico quanto com os materiais empregados na execução das habitações.

A adoção de aberturas maiores, melhor orientação e distribuição dos residenciais como um todo, além do plantio de vegetação nos residenciais, são modificações favoráveis à melhoria no conforto térmico das mesmas.

Devido às falhas no projeto arquitetônico desse tipo de edificação, verifica-se um grande número de ampliações e modificações, por parte dos moradores, após a ocupação dos imóveis, sobretudo, nos Residenciais Vila América e Sebastião de Matos I. Isso indica, de certa forma, a insatisfação do público com o produto final. Assim, é necessário que os projetos das HIS sejam revistos e readequados para o clima da região, de modo que possam propiciar mais conforto aos seus usuários.

\section{CONSIDERAÇÕES FINAIS}

O presente trabalho serviu para documentar o conhecimento disseminado entre os profissionais da área e confirmar a hipótese levantada: a inadequação das HIS para o clima do município de Sinop. Espera-se que os resultados alcançados possam servir como diretrizes norteadoras para a melhoria da qualidade das futuras HIS na região.

A obtenção dos dados para a realização da pesquisa mostrou-se um tanto dificultosa, sendo necessárias diversas tentativas de contato com as empresas, por diferentes meios. Mesmo assim, entende-se que a amostra analisada foi representativa, devido às semelhanças das tipologias e à pouca diferença entre os resultados obtidos.

Como sugestão para trabalhos futuros, propõe-se a avaliação das demais características relacionadas ao conforto propiciado pelas edificações, como, por exemplo, características acústicas, de 
luminosidade, entre outras, com vistas ao que preconiza a NBR 15575:2013 - Edificações Habitacionais - Desempenho.

\section{REFERÊNCIAS}

ASSOCIAÇÃO BRASILEIRA DE NORMAS TÉCNICAS - ABNT. NBR 15220 - Desempenho térmico em edificações. Rio de Janeiro: ABNT, 2005.

NBR 15575 - Edificações habitacionais - Desempenho. Rio de Janeiro: ABNT, 2013.

CÂMARA BRASILEIRA DA INDÚSTRIA DA CONSTRUÇÃO - CBIC. Desempenho de edificações habitacionais: guia orientativo para atendimento à norma ABNT NBR 15575/2013. Fortaleza: Gadioli Cipolla Comunicação, 2013.

HARRIS, A. L. N. C. Metodologias Baseadas na Teoria dos Sistemas Nebulosos (Fuzzy Systems Theory) no Tratamento as informações Subjetivas do Projeto Arquitetônico. 1999. Tese (Doutorado). Departamento de Engenharia de Construção Civil. Escola Politécnica da Universidade de São Paulo. 160 p. São Paulo, SP.

INSTITUTO BRASILEIRO DE GEOGRAFIA E ESTATÍSTICA - IBGE. Cadastro de Localidades Brasileiras Selecionadas. Disponível em: $\leq \mathrm{ftp}$ //geoftp.ibge.gov.br/organizacao territorial/localidades/Acesso em 02 out. 2013.

INSTITUTO DE PESQUISA ECONÔMICA APLICADA - IPEA. Nota Técnica: Estimativas do déficit habitacional brasileiro (2007 - 2011) por municípios (2010). Brasília: IPEA, 2013.

LEÃO, M. Desempenho Térmico em Habitações Populares para Regiões de Clima Tropical: Estudo de Caso em Cuiabá-MT. 2006. Dissertação. (Mestrado). Departamento de Física. Instituto de Ciências Exatas e da Terra. Universidade Federal de Mato Grosso. 105 f. Cuiabá, MT.

MAITELLI, G. T. Interações atmosfera-superfície. In: MORENO, G; HIGA, T. C. S. (Org.). Geografia de Mato Grosso: Território, sociedade, ambiente. Cuiabá: Entrelinhas, 2005. Cap. 13, p. 238-249.

MENEZES, M. S. Avaliação do desempenho térmico de habitações sociais de Passo Fundo - RS. 2006. Dissertação (Mestrado). Faculdade de Engenharia e Arquitetura. Universidade de Passo Fundo. Passo Fundo, RS.

ROTTA, R. Desempenho Térmico de Edificações Multifamiliares de Interesse Social em Conjuntos Habitacionais na Cidade de Santa Maria - RS. 2009. Dissertação (Mestrado). Programa de Pós-Graduação em Engenharia Civil. Centro de Tecnologia. Universidade Federal de Santa Maria. Santa Maria, RS.

SENA, C. B. Análise Comparativa entre o Método de Mahoney Tradicional e o Método de Mahoney Nebuloso para Caracterização do Clima no Projeto Arquitetônico. 2004. Dissertação (Mestrado). Departamento de Engenharia de Construção Civil. Escola Politécnica da Universidade de São Paulo. 132 p. São Paulo, SP. 